\title{
DER FÜR DAS SEELSORGLICHE WIRKEN DER KIRCHE IN ÖSTERREICH DURCH DAS STAATLICHE RECHT VORGEGEBENE RAHMEN
}

1. Das Österreichische Staatskirchenrecht setzt sich aus den Bauelementen verschiedenster Epochen zusammen, aber sehr entscheidend und grundlegend für seine heutige Gestalt war die unter den Impulsen der Revolution von 1848 stehende Gesetzgebung der zweiten Hälfte des vorigen Jahrhunderts. Von besonderer Bedeutung ist dabei:

- das Staatsgrundgesetz über die allgemeinen Rechte der Staatsbürger vom 21. Dezember 1867 (RGBL Nr. 142), dessen Grundrechtskatalog bis heute Bestandteil der österreichischen Verfassung ist. Dieses Staatsgrundgesetz gewährt

— die individuelle Religionsfreiheit des Einzelnen (Art. 14)

- und die kooperative Religionsfreiheit für die gesetzlich anerkannten Kirchen und Religionsgesellschaften (Art. 15)

2. Der Freiheitsraum, den das österreichische Recht der Entfaltung des Gewissens gewährt: Art. 14 StGG 1867 gewährleistet die volle Glaubens und Gewissensfreiheit. Spätere Dokumente, der Staatsvertrag von St. Germain (StGBl. 303/1920 Art. 63) und die Europäische Menschenrechtskonvention (RGBl. 210/1958 Art. 9), die Bestandteil des österr. Verfassungsrechtes sind, unterstreichen dies und weiten dies (insbesondere die Menschenrechtskonvention) noch im Sinne der Weltanschauungsfreiheit aus. In der historischen Entwicklung war die Katholische Religion Staatsreligion. Bei der großen Glaubensspaltung war der Protestantismus in Österreich nich zum Zuge gekommen, zum Teil war er mit Waffengewalt niedergeschlagen worden, aber er lebte in manchen Bereichen als Geheimprotestantismus fort. Es gab mancherorts Privilegien für einzelne Stände bzw. Kronländer, insbes. vom Kaiser, wenn er Steuern brauchte, (z.B. dem Adel) zum Privatexerzitium aber nicht zur öffentlichen Religionsübung gewährte, es gab auch Sonderprivilegien für Juden. Auch das Protestantenpatent 1781 gab nur das Privatexerzitium frei. Erst in der Folge der Revolution von 1848 weitete sich der Grundrechtsschutz aus. Im zitierten Staatsgrungesetz ist die individuelle Religionsfreiheit garantiert.

*Vortrag gehalten an der Päpstlichen Theologischen Akademie Krakau am 27. Februar 1986. 
3. Die korporative Religionsfreiheit im SEGG 1867: Sie wurde den gesetzlich anerkannte Kirchen und Religionsgesellschaften gewährt. Dies war mit dem Verlust der Stellung der Katholischen Kirche als Staatskirche verbunden. Die Katholische Kirche war nunmehr eine von den gesetzlich anerkammten Religionsgemeinschaften. Und es herrscht in bezug auf diese der Grundsatz Der Parität, der Gleichheit der gesetzlich anerkannten Konfessionen.

Der deutsche evangelische Staatskirchenrechtler Martin Heckel ${ }^{1}$ stellte in einem Vortrag vor der Heidelberger Akademie der Wissenschaften in allgemeiner Weise fest: Der Liberalismus hat, um das „Weg von der Kirche” zu gewährleisten, die Religionsfreiheit in der Verfassung verankert, aber die Religionsfreiheit ist nicht nur eine Freiheit zum Unglauben sondern auch eine Freiheit zum Glauben. Die Katholische Kirche habe dies rascher erkannt als die Evangelische und nicht so sehr den Verlust der Stellung als Staatskirche beklagt, sondern die Freiheit der religiösen Gestaltung für sich in Anspruch genommen. Sie hat versucht, die staatliche Bevormundung, die ja mit der Stellung als Staatskirche - zumindest bis zur Revolution von 1848 gegeben warabzuschütteln.

4. Vom Jahre 1867 (vom zit. StGG) datiert auch die Unterscheidung in gesetzlich anerkannte und gesetzlich nicht anerkannte Religionsgemeinschaften $^{2}$. Nur die gesetzlich anerkannten Konfessionen hatten das Recht der öffentlichen Religionsübung, die anderen nur das der privaten, (keine öffentlichen prozessionen, keine Glocken, die Gotteshäuser durften nicht als solche nach außen gekennzeichnet sein). In der Zwischenzeit wurde übrigens durch den Staatsvertrag von St. Germain (Art. 63 Abs. 2) allen Einwohnern Österreichs, und so auch den Anhängern gesetzlich nicht anerkannter Religionsbekenntnisse die öffentliche Religionsübung zugestanden. Die Sonderstellung der gesetzlich anerkannten Konfessionen war aber auch noch anders begründet. Der Staat wollte die Kirchen durch die gesetzliche Anerkennung unter Kontrolle haben ${ }^{3}$. Es wirkten dabei auch josephinische Tendenzen des Staatskirchentums - zumindest unbewußt - nach.

5. Die gesetzlich anerkannten Kirchen und Religionsgesellschaften galten und gelten bis heute als öffentliche Corporationen. Sie sind nicht private Vereine sondern öffentlichen Rechts. Ihnen steht die öffentliche Schule für den RU offen. Für ihre seelsorglich Tätigen gilt nicht das Arbeitsverfassungsrecht, das sonst in Betrieben eine weitgehende Mitsprache von Betriebsräten vor-

${ }^{1}$ M. Hecke l, Korollarien zur Säkularisierung, Heidelberg 1981 (Sitzungsberichte der Heidelberger Akademie der Wissenschaften, Philosophischhistorische Klasse 1981/4).

2 Terminologisch ist zu sagen, daß das österreichische Staatskirchenrecht den Ausdruck Kirchen und Religionsgesellschaften verwendet, weil eben nicht alle Religionsgemeinschaften Kirchen sind, z.B. die Religionsgesellschaft der Anhänger des Islam.

${ }^{3}$ Die Tendenzen des damaligen Liberalismus waren ja nicht nur kirchenfreundlich. Das nach Hinfälligerklärung des 1855 er Konkordates durch Österreich (1870) erlassene Katholikengesetz legte der katholischen Kirche eine stärkere Bevormundung auf, als anderen gesetzlich anerkannten Kirchen. 
sieht usw. Der Grund, auf dem ein Gotteshaus steht, ist grundsteuerbefreit. Die seelsorgliche Tätigkeit (Predigt usw. Aushilfen) ist wie die Verwaltungstätigkeit der staatlichen Behörden, mit der sie verglichen wird, umsatzsteuerfrei. Kirchen und ihre Einrichtungen zahlen Körperschaftssteuer nur für gewerbliche Betriebe, die sie führen, sie sind diesbezüglich den Staat gleichgestellt. Das geistliche Amtsgeheimnis - nicht nur das Beichtgeheimnis - ist vor Gericht in einer dem Amtsgeheimnis der Staatsbeamten vergleichbaren Weise geschützt. Wird ein Geistlicher als Zeuge einvernommen, so kann er vom Richter nicht über das befragt werden, was der geistlichen Verschwiegenheitspflicht unterliegt. Ein entsprechender verfahrensrechtlicher Schutz besteht im Strafprozeß, im Zivilprozeß, im Verwaltungsverfahren, im Verfahren nach der Bundesadgabenordnung (Steuern usw.), im Verfahren vor dem Verfassungs- und vor dem Verwaltungsgerichtshof.

6. Die öffentlich-rechtliche Stellung, die den Kirchen eignet, führt also weitgehend zu Parallelen ${ }^{4}$ mit dem Staat und mit staatlichen Körperschaften, wie Kammern oder Gemeinden. Doch sind die Kirchen diesen nicht in allem gleichgezogen. Während Gemeinden und staatliche Körperschaften für ihr gesamtes Tun an die Grundsäbe der österreichischen Bundesverfassung gebunden sind und der Kontrolle der Gerichtshöfe des öffentlichen Rechtes (Verfassungsgerichtshof, Verwaltungsgerichtshof) unterliegen, sind die Kirchen und Religionsgesellschaften in dem, was das österreichische Staatskirchenrecht die ,inneren Angelegenheiten" nennt ${ }^{5}$ (Glaubenslehre, Kult, Organisation usw.), frei, es ist dies eine Sphäre, in die der Staat nicht ingeriert.

7. Es gibt natürlich auch Angelegenheiten der Kirche(n), die so nach außen wirken, daß sie mit dem staatlichen Leben verbunden sind. So z.B. der Religionsunterricht in der Schule, die Militärseelsorge, die Anstaltsseelsorge, (in Krankenanstalten, Gefängnissen usw.), die theologischen Fakultäten an staatlichen Universitäten und hier gibt es eben Regelungen mit dem Staat.

8. Dabei ist zu beachten: der Freiheitsbegriff, der der österreichischen Verfassung zugrundeliegt, gewährleistet auch die freie Gestaltung des Lebens der einzelnen anerkannten Religionsgemeinschaften nach jeweils ihrem Selbstverständnis. Darum sind die Berührungen mit dem Staat nicht immer ganz gleich. Der Gleichheitssatz der österreichischen Bundesverfassung verlangt, das Gleiches gleich und Ungleiches ungleich behandelt wird. Es widerspricht also der Gleichbehandlung nicht, wenn der Staat auf die einzelnen Kirchen nach jeweils ihrem Selbstverständnis eingeht und so mit der Katholischen Kirche ein Konkordat schließt (völkerrechtlicher Vertrag), für die Protestanten hat er nach Absprache mit der evangelischen Kirchenleitung ein eigenes, rein inner-

${ }^{4}$ Der Mißbrauch des geistlichen oder des Ordensgewandes (das Tragen durch Laien) wird in gleicher Weise wie der Mißbrauch militärischer Uniformen bestraft (Art. XXI Konkordat).

${ }^{5}$ Vgl. hiezu: H. S c h we n d e n w e in, Das neue Kirchenrecht Gesamtdarstellung, Graz-WienKöln 1984, S. 57. 
staatliches Gesetz erlassen: das Protestantengesetz, für die Orthodoxen das Orthodoxengesetz. Aus der Zeit der Monarchie gelten noch das Israelitengesetz und das Islamgesetz.

9. Das österr. Konkordat vom 5. Juni 1933, von manchen auch nach dem Inkrafttreten als Konkordat vom 1. Mai 1934 bezeichnet, wird heute allgemeinals zu Recht bestehend und gültig anerkannt. Seine Normen gelten innerstaatlich wie die eines einfachen Bundesgesetzes. Beispielsweise wurde, wenn einem Professor einer theologischen Fakultät das kirchliche Placet entzogen wurde, dieser durch den Bundesminister für Wissenschaft und Forschung gemäß Art. $5 \S 4$ Abs. 1 Konkordat von der Ausübung der Lehrtätigkeit enthoben und gemäß dem Zusatzprotokoll zu Art. V $\S 4$ des Konkordates in den Ruhestand versetzt. Nur wenige Bestimmungen des Konkordates sind durch einen späteren Vertrag zwischen dem Hl. Stuhl und der Republik Österreich, den sogenannten Vermögensvertrag von 1960, aufgehoben worden.

10. Es gibt zwischen dem $\mathrm{Hl}$. Stuhl und der Republik Österreich außer dem Konkordat von 1933 (1934) noch Verträge, die nur einzelne Tailbereiche der Rechtsbeziehungen regeln, so den eben erwähnten Vermögensvertrag von 1960, den Schulvertrag von 1962 und die Verträge über die Errichtung der Diözesen: Eisenstadt 1960, Innsbruck 1964 (Innsbruck-Feldkirch; ab 1968 Insbruck) und Feldkirch (1968).

11. Die Errichtung neuer Diözesen bedarf wie jede größere Änderung der territorialen Diözesanorganisation des Einvernehmens mit der Staatsgewalt (Art. III § 1 Konkordat). Übrigens werden in den erwähnten Diözesanerrichtungs verträgen auch finanzielle Hilfen seitens der Staatsgewalt gewährt.

12. Die Ernennung der Bischöfe erfolgt frei durch den Hl. Stuhl, mit Ausnahme des Erzbischofs von Salzburg: Im Falle der Vakanz des erzbischöflichen Stuhles von Salzburg benennt der Hl. Stuhl dem Metropolitankapitel drei Kandidaten, aus welchen dieses ,in freier, geheimer Abstimmung den Erzbischof” wählt (Art. IV § 1 Abs. 3 Konkordat). Im übrigen bestimmt Art. 4 § 2 des Konkordates, daß vor der Ernennung residierender Bischöfe (Diözesanbischöfe) oder von Koadjutoren der Hl. Stuhl den Namen des in Aussicht Genommenen der Bundesregierung mitteilen wird, um zu erfahren, ob sie Gründe allgemeiner politischer Natur gegen die Ernennung geltend zu machen hat. Im Falle der Nichtbeantwortung kann der Hl. Stuhl nach 14 Tagen zur Ernennung schreiten. Die Regierung kann also nur aus Gründen allgemeinpolitischer Natur, nicht aus Gründen parteipolitischer Natur Bedenken erheben ${ }^{6}$. Es gibt ja vergleichbare Klauseln auch in anderen Konkordaten und es gibt darüber - in Deutschland - auch einige Literatur.

13. In diesem Zusammenhang darf gleich auch angemerkt werden, daß der

${ }^{6}$ Vgl. hierzu: R. Köstle r, Das neue österreichische Konkordat, „Zeitschrift für Öffentliches Recht"1935, S. 11. 
Kirche der freie Verkehr mit Rom und die freie Ausübung innerkirchlicher Gesetzgebung, der Vollzug des Kultus und der Seelsorge garantiert ist (Art. I Konkordat). Dies entspricht ja auch dem obzitierten Art. 15 StGG, der die Freiheit in den inneren Angelegenheiten gewährleistet.

14. Bezüglich des obgenannten Vermögensvertrages möchte ich hier folgendes herausheben. Es wurde der Versuch unternommen, eine gewisse Entschädigung für die der Kirche unter dem Nationalsozialismus zugefügten Schäden zu geben. Damals ist ja nicht nur kirchliches Eigentum beschlagnahmt worden, das nach 1945 wieder zurückgegeben werden mußte, die Kirche hat auch Einnahmequellen verloren, so wurden vor der nationalsozialistischen Ära aus den staatlich verwalteten Religionsfonds, die aus dem Vermögen der von Josef II (1780-1790) aufgehobenen Klöster gebildet worden sind, weitgehendst die kirchlichen Ausgaben bestritten, insbesondere der Klerus besoldet. Es gab dazu noch Staatszuschüsse, damit die geistlichen Amtsträger eine angemessene Besoldung, die Kongrua, erhalten konnten. Dies fiel unter der deutschen Besetzung weg. Im Vermögensvertrag von 1960 wurden der Kirche einige Vermögensobjekte gegeben und außerdem ein laufender (jährlicher) Staatszuschu $\beta^{7}$, der bis heute bezahlt wird. Er reicht natürlich nicht aus, um die ganze Klerusbesoldung und überhaupt den Kirchenaufwand zu bestreiten. D.h., die Kirche braucht noch weitere Einnahmequellen, auch die Erträge kirchlichen Vermögens bringen nicht genug, es ist heute überhaupt schon schwer, vom Ertrag des Vermögens zu leben.

15. Bis zur Zeit der deutschen Besetzung waren die Österreicher abgesehen von Almosen, z. B. bei der Sammlung im Sonntagsgottesdienst, - nicht gewohnt, für den kirchlichen Bedarf, der ja, wie gesagt, weitgehend aus öffentlichen Mitteln (Religionsfonds usw.) bestritten wurde, aufzukommen. Die Nationalsozialisten glaubten, durch Entzug der staatlichen Finanzierung und Einführung einer Art Kirchensteuer, der Kirchenbeiträge, durch welche die Katholiken die ganzen Kosten des Kirchenaufwandes tragen mußten, eine Kirchenaustrittswelle auszulösen. Diese Erwartungen haben sich nicht erfüllt. Gerade die Bedrohung und Verfolgung hat die Katholiken zusammengeschweißt und in ihnen die Bereitschaft, für die Kirche auch finanzielle Opfer zu bringen, aktiviert. Zu keiner anderen Zeit, als in der Zeit der Verfolgung wäre die Einführung einer solchen Belastung möglich gewesen.

Da wie gesagt, auch die Wiedergutmachungsmaßnahmen nach 1945 nicht das zur Gänze ausgeglichen haben, was die staatliche bzw. öffentliche Kirchenfinanzierung bis 1938 bzw. 39 brachte, mußte man, um den Bedarf der Kirche zu decken, die Kirchenbeiträge, durch welche die volljährigen Kirchenangehörigen je nach ihrem Einkommen eine jährliche Abgabe an die Kirche lei-

${ }^{7}$ Bestehend aus einem Nominalbetrag und dem Gegenwert von 1250 Beamtengehältern der Verwendungsgruppe A Dienstklasse 4,4. Gehaltsstufe. 
sten, beibehalten. Im Lichte der heutigen Verfassungsordnung können die Kirchen von ihren Gläubigen, ohne daß es hiezu eines eigenen staatlichen Gesetzes bedarf, Beiträge, oder Umlagen einheben. Dies ist eine innerkirchliçhe Angelegenheit gemäß Art 15 Staatsgrundgesetz. Einer besonderen staatlichen Rechtsgrundlage bedarf es, wenn der Staat seinen Beistand zur Einhebung gewähren soll. Und als solche wird das Kirchenbeitragsgesetz von 1939 verstanden. Die Kirchenbeitragsordnungen der einzelnen Diözesen unterliegen der staatlichen Genehmigung, dafür kann, wenn ein Gläubiger der Kirchenbeitragszahlung nicht nachkommt, dieser vor dem staatlichen Gericht geklagt werden.

16. $\mathrm{Zu}$ beachten ist noch, daß das Konkordat den kirchlichen juristischen Personen auch staatliche Rechtspersönlichkeit zuerkennt (Art. II, X § 2, XV § $7-22$. Satz). Allerdings ist bei neu entstehenden kirchlichen Rechtsträgern notwendig, daß sie der Diözesanordinarius der obersten staatlichen Kultusbehörde meldet. Juristische Personen der Katholischen Kirche können nach dem Konkordat frei Vermögen erwerben (Art. XIII § 1). Von der Österreichischen Verfassungsordnung her hätte der einfache Bundesgesetzgeber die Möglichkeit, Erwerbsbeschränkungen der Toten Hand festzulegen (Art. VI Abs. 2 Staatsgrundgesetz), doch hat sich Österreich im Konkordat, also in einem völkerrechtlichen Vertrag verpflichtet, von dieser Möglichkeit nicht gegen die katholische Kirche Gebrauch zu machen ${ }^{8}$. Das Konkordat und damit auch die Norm, nach welcher kirchliche juristische Personen in Österreich Vermögen frei erwerben können (Art. XIII § 1), ist nicht nur staatliches sondern auch kirchliches Recht. D.H. also, daß auch die Mendikantenklöster nach österreichischem Partikularkirchenrecht voll vermögens- und erwerbsfähig sind. Allfällige nach ihrem jeweiligen Ius Proprium sonst bestehende Beschränkungen bestehen seit dem Inkrafttreten des Konkordates, seit dem 1. Mai 1934 in Österreich nicht mehr. Auch die Kapuziner sind nunmehr Eigentümer ihrer Klöster.

17. Ein sehr wichtiger das Konkordat ergänzender Vertrag zwischen dem Hl. Stuhl und der Republik Österreich ist der Schulvertrag von 1962, der den Religionsunterricht und die Katholischen Schulten betrifft. Die Bestimmungen des nur für die katholische Kirche geltenden Schulvertrages decken sich weitgehend ${ }^{9}$ mit denen des Religionsunterrichtsgesetzes, eines staatlichen für alle gesetzlich anerkannten Kirchen und Religionsgesellschaften geltenden Gesetzes und bieten so für die Katholische Kirche praktisch eine zusätzliche, nämlich eine völkerrechtliche Absicherung des Religionsunterrichtes. Reli-

${ }^{8}$ H. Schwendenwein, Erwerbsbeschränkungen der Toten Hand in Österreich, AKKR 92:1973 S. $455-462$.

9 Bezüglich der Unterschiede vgl.: H. Schwendenwein, Verfassung, Religionsunterrichtsgesetz und Schulvertrag, „Christlich-Pädagogische Blätter” 92:1979 S. 135-136. 
gionsunterricht ${ }^{10}$ ist an nahezu allen Schultypen für Schüler, die einer gesetzlich anerkannten Kirche oder Religionsgesellschaft angehören, Pflichtfach mit Abmeldungsmöglichkeit ${ }^{11}$, d.H., wenn zu Beginn des Schuljahres keine Ambeldung durch die Eltern oder (bei bereits 14 jährigen) durch den Schüler erfolgt, ist Religion für ihn verpflichtend. Religion darf nur unterrichten, wer die kirchliche (religionsgesellschaftliche) Befähigung und Ermächtung hat. Die Religionslehrer werden vom Staat besoldet. Bei Entzug der kirchlichen Ermächtigung darf der betraffende für den Religionsunterricht nicht mehr verwendet werden. Für Inhalt und Methode des Religionsunterrichtes ist die Kirche zuständig, der Religionslehrer wird diesbezüglich von kirchlich bestellten Inspektoren inspiziert, in Fragen der Schuldisziplin und Schulorganisation untersteht er den für alle Lehrer zuständigen Landes - bzw. Bezirksschulinspektoren. Das österreichische Religionsunterrichtsrecht ist streng konfessionell orientiert, jede gesetzlich anerkannte Kirche oder Religionsgesellschaft hat ihren eigenen Religionsunterricht, der bei hinreichender Schülerzahl auch staatlich finanziert wird. Lediglich in der Frage der Schulkreuze ist nicht auf einzelne Konfessionen abgestimmt: wenn die Mehrheit der Schüler einer Klasse einem christlichen Bekenntnis angehört, so ist in der betreffenden Klasse ein Kreuz anzubringen. Die Kirche hat auch die Möglichkeit, katholische Privatschulen $^{12}$ mit Öffentlichkeitsrecht zu errichten. Die Zeugnisse einer Schule mit Öffentlichkeitsrecht sind wie die Zeugnisse einer öffentlichen (einer staatlichen) Schule anerkannnt. Voraussetzung ist natürlich die Gleichwertigkeit mit den öffentlichen Schulen (insbes. diplomierte Lehrer). Das katholische Schulwesen, das den Anforderungen entspricht und eine Entlastung des öffentlichen Schulwesens darstellt, wird vom Staat, der $100 \%$ des Personalaufwandes trägt, unterstützt. Die staatliche Subventionierung erfolgt durch Zuweisung „lebender Subventionen”, d.h. durch Zuweisung staatlich angestellter und besoldeter Lehrer zur Dienstleistung, wobei die Kirche die Möglichkeit hat, Lehrer abzulehnen. In Ordensgemeinschaften gehörigen Schulen wird für ordenseigene Lehrkräfte eine staatliche Entschädigung gezahlt.

18. Österreich hat vier staatliche Katholisch-Theologische Fakultäten (an den Universitäten Wien, Graz, Salzburg und Innsbruck). Österreich anerkennt die Akademischen Grade der päpstlichen Universitäten in Rom ${ }^{13}$. Im übrigen sind ja für die Anerkennung ausländischer Grade die Fakultätskolle-

${ }^{10}$ Des Näheren siehe: H. Schwendenwein, Religion in der Schule. Rechtsgrundlagen, Graz 1980; derselbe, Der Religionsunterricht im österreichischen Recht, ,Theologisch-praktische Quartalschrift" 127:1979 S. 376-381.

${ }^{11}$ Siehe hiezu: H. Schwendenwein, Religion als Pflichtfach, „Christlich-Pädagogische Blätter” 92:1979 S. 134-136; derselbe, The Training of Catechists in Austria, „Studia Canonica” 15:1981 S. $241 \mathrm{f}$.

12 Vgl. hiezu: H. Sch w e n de n w e in, La escuela privada confesional en el Derecho Austriaco, „Revista Española de Derecho Canonico” 37:1981 S. 445-449.

"Ri3 Art. V Konkordat. Auch die Grade der päpstlichen Fakultät Linz sind in Österreich anerkannt. 
gien zuständig. Niemand kann an den österreichischen theologischen Fakultäten lehren, der nicht das Placet des zuständigen Bischofs hat (Art. V § Konkordat). Im übrigen erfolgt die Bestellung der Hochschullehrer genau so wie an den anderen Fakultäten. Vor Bestellung von Assistenten und Professoren, vor Erteilung von Lehraufträgen und vor Bestätigung einer Habilitation durch den Bundesminister wird das bischöfliche Placet eingeholt. Wird dieses später entzogen, so ist der Betroffene von der Lehrtätigkeit an den theologischen Fakultäten zu entheben (Art. V § 4 Konkordat). Der österreichische Staat gibt Studienvorschriften für die Theologie in Anlehnung an die kirchlichen Normen ${ }^{14}$.

19. Österreich hat eine konkordatorisch verankerte Militärseelsorge. Der Militärvikar, der die bischöfliche Würde bekleiden muß (Art. VIII § 1 Abs. 2 Konkordat), wird kirchlich vom $\mathrm{Hl}$. Stuhl bestellt, nachdem dieser sich bei der Bundesregierung in vertraulicher Form unterrichtet hat, ob gegen die in Aussicht genommene Persönlichkeit allgemein politische Bedenken vorliegen (Art. VIII § 1 Abs. 1 Konkordat). „Die kirchliche Bestellung der Militärkapläne erfolgt zunächst durch den Militärvikar nach vorherigem Einvernehmen mit dem Bundesminister für Heerwesen" (Art. VIII § 2 Konkordat). Erst auf dieser Grundlage wird die staatliche Ernennung vorgenommen. Die Militärsgeistlichen tragen Offiziersuniform.

20. Selbstverständlich haben die zuständigen Ortsseelsorger oder sonstige hiezu kirchlich beauftragte Seelsorger freien Zutritt in öffentliche (staatliche) Spitäler, Heil-, Versorgungs- und dergleichen Anstalten, in Gefangenenhäusern, Strafanstalten, Anstalten für Erziehungsbedürftige und dgl. „behufs freier Ausübung” des „geistlichen Amtes” an den "Anstaltsinsassen” (Art. XVI Konkordat). Manche Anstalten, z.B. das Grazer Sonderkrankenhaus hat einen eigenen vom Spitalserhalter (in diesem Fall vom Land Steiermark) besoldeten, im Einvernehmen mit dem Bischof bestellten hauptamtlichen Seelsorger. Die österreichische Justizverwaltung hat mehrere hauptamtliche, staatlich besoldete Beamtenstellen für im Einvernehmen mit dem jeweiligen Bischof zu bestellende-Seelsorger an Strafvollzugsanstalten und Untersuchungsgefängnissen.

21. Geistliche und jene, die sich auf ein geistliches Amt vorbereiten, sind nach dem Wehrgesetz von der Stellungspflicht befreit, sie brauchen keinen Militärdienst zu leisten. Nach Art. XIX des Konkordates sind Geistliche und Ordenspersonen vom Geschworenen- und Schöffenamt befreit. Auch das beneficium competentiae, das noch im CIC $1917 \mathrm{zu}$ den Klerikerprivilegien gehört hat, findet seinen Niederschlag im Konkordat: Das Amtseinkommen von Geistlichen ist im gleichen Maße wie das der Angestellten des Bundes exeku-

${ }^{14} \mathrm{Vgl}$. hiezu: H. Schwende nwe in, Grundfragen der Entwicklung des theologischen Studienrechtes in Österreich seit Beginn des 20. Jahrhunderts, [in:] Domus Austriae. Eine Festgabe Hermann Wiesflecker zum 70. Geburtstag. Hrsg. von W. Höflechner, H. J. Mezler-Andelberg, O. Pick l, Graz 1983, s. 373-377. 
tionsfrei (Art. XVII Konkordat). Nur genießen eben nach derzeitigem österreichischem Recht Bundesangestellte als solche keinen exekutionsrechtlichen Schutz, der über den den einen geistigen Beruf persönlich Ausübenden gewährten hinausgeht. Für diesen Personenkreis und damit auch für Geistliche sind die zur Verwaltung des Dienstes oder Vorbereitung und Ausübung des Berufes erforderlichen Gegenstände, sowie die anständige Kleidung exekutionsfrei ( $\$ 251$ Ziffer 5 der Exekutionsordnung). Nach § 250 der Exekutionsordnung sind Gegenstände, die zur Ausübung des Gottesdienstes einer gesetzlich anerkannten Kirche oder Religionsgesellschaft verwendet werden, sowie Kreuzpartikel und Reliquien, mit Ausnahme der Fassung unpfändbar. Nach Art. XX des Konkordates hat das staatliche Gericht im Falle der strafgerichtlichen Belangung eines Geistlichen den für den Belangten zuständigen Diözesanordinarius zu verständigen und demselben raschestens die Ergebnisse der Voruntersuchung und gegebenenfalls das Endurteil des Gerichtes sowohl in der ersten als auch in der Berufungsinstanz zu übermitteln (Abs. 1). Im Falle der Verhaftung und Anhaltung in Haft soll der Geistliche (Ordensperson) mit der seinem Stande und der seinem hierarchischen Grade gebührenden Fücksicht behandelt werden (Abs. 2).

22. Es gibt auch einen strafrechtlichen Schutz der religiösen Sphäre. Im österreichischen Strafgesetzbuch (1974) finden sich eigens dem Schutz der Religion dienende Straftatbestände: Sachbeschädigung ( $\$ 126)$ und Diebstach ( $§$ 128 ) an Sachen, die dem Gottesdienst oder der Verehrung einer im Inland bestehenden Kirche gewidmet sind, bzw. an Grabstätten und Friedhöfen ( $\$ 126)$, Diebstahl an der Religionsausübung dienenden Örtlichkeiten (\$ 128)? Störung der Totenruhe ( $\$ 190)$, Herabwürdigung religiöser Lehren ( $\$ 188)$, Störung einer Religionsübung $(\$ 189)$. Natürlich können auch andere Straftatbestände dem Schutz des Religiösen zugute kommen, so z.B. die auf Verhetzung ( $\$$ 283 ), oder auf Sprengung ( $\$ 284)$, Verhinderung oder Störung einer Versammlung bezüglich $(\S 285)$.

23. Österreich hat ein ausgeprägtes, auch die Pension im Alter umgreifendes System der öffentlich-rechtlichen Sozialversicherung, von dem auch die Dienstnehmer - mit wenigen Ausnahmen - erfaßt sind. Ausgenommen von diesem System sind u.a. die im Dienst ihrer Kirchen stehenden katholischen und evangelischen Geistlichen, sowie Ordensleute, Angehörige von Kongregationen und von evangelischen Diakonissenanstalten ${ }^{15}$. Sie zahlen keine Sozialversicherungsbeiträge und erhalten später keine Pension aus der öffentlich-rechtlichen Pensionsversicherung der Angestellten. Grund dieser Ausnahme ist, daß für diese Personengruppen ohnedies eine innerkirchliche Altersversorgung besteht. Die im Diözesandienst stehenden Weltpriester erhalten nach der Emeritierung eine Pension aus dem Diözesanbudget, die Ordensleu-

${ }^{15}$ Vgl. H. Schwe nde nwe in, Rechtsfragen in Kirche und Staat, Graz 1979, S. 39 f. 
te haben die Altersversorgung im Orden. Im Falle des Ausscheidens aus dem geistlichen Stand wird der Betreffende für die Zeit, für die er im kirchlichen Dienst stand, in der Pensionsversicherung der Angestellten nachversichert.

24. Im Bereich der Beziehungen Kirche - Staat spielt auch der Denkmalschutz eine gewisse Rolle. Der Eigentümer eines als Denkmal qualifizierten Bauwerkes ${ }^{16}$ unterliegt einerseits bei baulichen Veränderungen großoben Einschränkungen in der Gestaltungsmöglichkeit — dies würde die Kirche, die ja die Erhaltung kultureller Werte begrüßt, nich so beeinträchtigen — und hat anderseits die Sorge für die entsprechende Erhaltung solcher Objekte, die bekanntlich kostenmäßig besonders aufwendig ist, wobei die Staatszuschüsse relativ gering sind. Während Bauwerke Eigentümer nur, wenn ein ausdrücklicher Bescheid für das betreffende Denkmal ergeht, unter Denkmalschutz stehen, unterliegen Denkmale, die im Eigentum öffentlich-rechtlicher Körperschaften, gesetzlich anerkannter Kirchen (Religionsgesellschaften) stehen, automatisch, gleichsam kraft Gesetzes dem Denkmalschutz, solange nicht auf Antrag des Eigentümers das Gegenteil festgestellt wird. So unterstehen kirchliche Baulichkeiten - auch Pfarrhöfe - in den allermeisten Fällen dem Denkmalschutz, was zwar die kulturelle Bedeutung der Kirche unterstreicht, sich aber gleichzeitig als zusätzliche finanzielle Belastung darstellt.

16 In praxi interessieren uns hier primär Bauwerke, obwohl das österreichische Recht den Denkmalschutz nicht auf Bauwerke beschränkt. 